\title{
A LOW-COST AND LIGHTWEIGHT 3D INTERACTIVE REAL ESTATE-PURPOSED INDOOR VIRTUAL REALITY APPLICATION
}

\author{
K.Ozacar ${ }^{\text {a, }}$, Y. Ortakci ${ }^{\text {a }}$, I. Kahraman ${ }^{\text {a }}$, R. Durgut ${ }^{\text {a }}$, I. R. Karas ${ }^{\text {a }}$ \\ ${ }^{a}$ Karabuk University, Computer Engineering Department, 78050 Demir Celik Campus, Karabuk, Turkey - (kasimozacar, \\ yasinortakci, idriskahraman, rafetdurgut, ismail.karas)@karabuk.edu.tr
}

KEY WORDS: Virtual Reality, Real-estate, Indoor, 3D Interaction, Navigation, Head-Mounted-Display

\begin{abstract}
:
Interactive 3D architectural indoor design have been more popular after it benefited from Virtual Reality (VR) technologies. VR brings computer-generated 3D content to real life scale and enable users to observe immersive indoor environments so that users can directly modify it. This opportunity enables buyers to purchase a property off-the-plan cheaper through virtual models. Instead of showing property through 2D plan or renders, this visualized interior architecture of an on-sale unbuilt property is demonstrated beforehand so that the investors have an impression as if they were in the physical building. However, current applications either use highly resource consuming software, or are non-interactive, or requires specialist to create such environments. In this study, we have created a real-estate purposed low-cost high quality fully interactive VR application that provides a realistic interior architecture of the property by using free and lightweight software: Sweet Home 3D and Unity. A preliminary study showed that participants generally liked proposed real estate-purposed VR application, and it satisfied the expectation of the property buyers.
\end{abstract}

\section{INTRODUCTION}

Virtual Reality (VR) is one of the most popular technology that has been currently used in real estate. Today, many people purchase a property off-the-plan as an investment opportunity. Because they hope the unbuilt property will worth more when it is built. Therefore, they can purchase a property less than its real value. However, purchasing a property off-the-plan has some limitations such as not being able to imagine the completed architecture of the property for the investors. In addition, the construction that built of this plan may not satisfy the expectation of the property investor when it is fully built. Consequently, purchasing a property through a $2 \mathrm{D}$ plan causes undoable investment plan.

To overcome these limitations, the interior architecture of an on-sale property must be demonstrated beforehand so that the investors have an impression as if they were in the building. Even though, there exists numerous ways to realize such scenario, $360^{\circ}$ panoramic and $3 \mathrm{D}$ immersive visualization.

Regarding panoramic visualization, there is a wide range of applications in which users navigate on a structure using a set of predefined paths. If the user wants to observe beyond the visualized part of the structure, the system will not respond and user will not see the other details that is not presented in the application. Besides, to provide panoramic view, the property sellers have to construct a demonstrative real property. It costs money and time for the sellers.

However, using 3D immersive visualization does not have such a limitation since it real-time renders the interior of a structure prospectively. Therefore, it allows users not only to visualize interior and walk along it, but also possibly to change the its properties according to the user's demands such as changing flooring, wall colours and so on. However, creating such a virtual environment requires some specialists and highly resource consuming software such as SketchUp, 3ds Max, Blender, etc.

In this study, we have created a real-estate purposed low-cost high quality fully interactive VR application that provides a realistic interior architecture of the property. We used the free and lightweight software: Sweet Home 3D and Unity. Former extracts 3D interior design model of the building from the 2D floorplans, latter enables us to create a fully interactive 3D immersive virtual environment. A user can walk through the indoor environment and observe the interior architecture including furniture, floor, walls etc. from different point of views. It gives the users confidence to decide to purchase a property off-the-plan. In addition to free and lightweight software, the only equipment we need to realize such application is a VR headset that allows us to feel as if we are wandering in a fully architectured building with realistic interior views.

\section{RELATED WORK}

There have been many studies, which each has its own strength and weakness, regarding interior architectural design in VR environment.

(Deaky and Parv, 2017) made a virtual interior architectured model of a real estate-purposed house in Unity 3D game engine in order to save property sellers' and buyers' time and money. Their interactive $360^{\circ}$ VR application works on various HMD (Head-Mounted Display) devices such as Google Cardboard and Oculus Rift. Even though, they have achieved a realistic interactive environment, there is no information regarding whether the software they utilized is free or lightweight.

\footnotetext{
* Corresponding author
} 
(Pejić et al., 2017) created an Augmented Reality (AR) and VR applications to present interior and exterior architectural projects. The user can interactively observe $360^{\circ}$ spherical panoramic images of the model by using AR and VR devices. The users can only navigate on a set of predefined paths in the indoor environment.

(Siltanen and Oksman, 2013) developed an AR interior home planner for different target user groups. User can insert, move and remove virtual furniture in a physical room through a mobile device with camera. They aimed to provide a design concept of an interior house to these target groups.

(Mudliyar et al., 2014) created a simple virtual reality system using OpenGL to visualize indoor environment for design in VR. However, their application does not allow users to modify indoor environment and lacks of realistic view as well.

(Xiao Yu, 2011) presented an interactive VR indoor environment for real-estate by using 3DS MAX for modelling and Virtools for interaction. Even if the users can modify the walls and probes, and turn on/off the lights in a room, the application was made in paid and highly resource consuming software.

(Vosinakis et al., 2007) presented web-based interactive application in which non-expert users select furniture and decorate elements, and try various arrangements in a room. To reduce the loading duration since it is a web-based application, their system only allows users to manipulate low-poly objects in one single room.

(Shaoliang Qi, 2012) created a semi-interactive virtual interior design of a house by using 3ds MAX and VRML to allow users observing indoor environment. His application is not completely interactive since passing from one room to another one is done by selecting the room name from a list displayed on the screen. In addition, the application was developed in commercial and heavyweight software.

There are many studies about VR application that visualize the indoor environment of a construction in the literature. However, they either lack the interaction sufficiently, or are designed in commercial and heavyweight software, or do not present realistic 3D view. Our application was developed in completely free, user-friendly and lightweight software as fully $3 \mathrm{D}$ interactive real-time VR application.

\section{INTERACTIVE REAL-ESTATE PURPOSED VR APPLICATION}

To create 3D virtual environment, we have used free and lightweight software.

\subsection{Creating 3D models using 2D interface}

We have used Sweet Home 3D home design software. This free software with its simple split screen $2 \mathrm{D} / 3 \mathrm{D}$ views one can draw walls and room in 2D window, and simultaneously visualize them in 3D view (Figure 1). It also let novice users to click on the object to easily change the texture, colour or material so it matches their design. In addition, the user can drop objects into the $2 \mathrm{D}$ design and then drag them to desired place within the design.

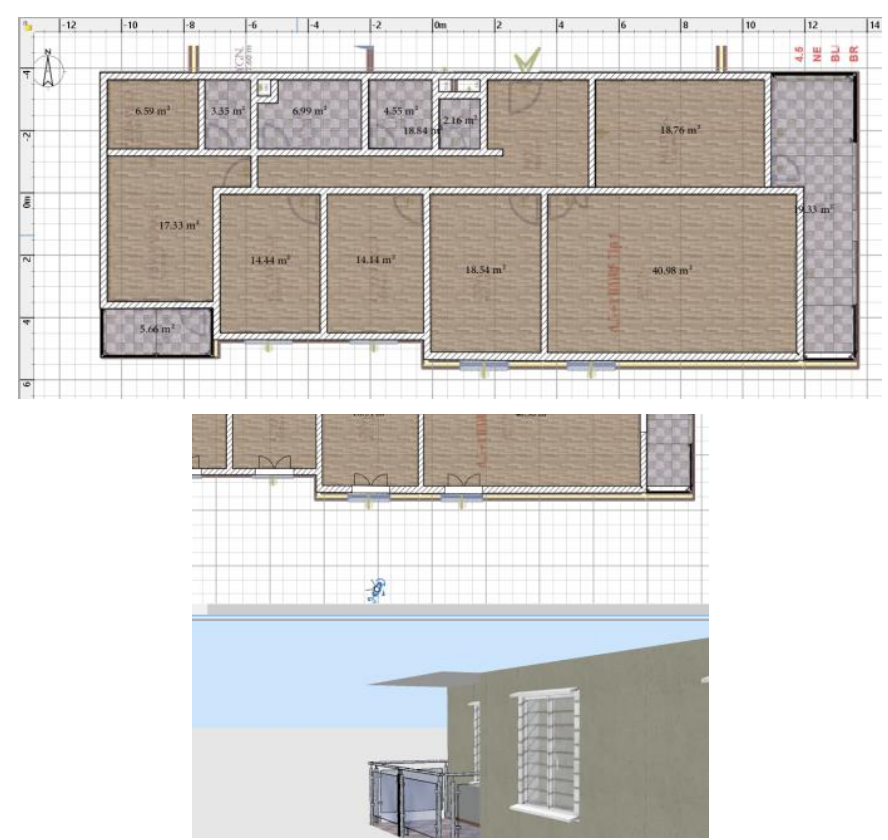

Figure 1. 2D floor plan (upper), and its 3D view with split screen in Sweet Home 3D. (bottom)

\subsection{Creating Interactive and Realistic 3D Environment}

Unity $3 \mathrm{D}$ is a cross-platform free game engine and is designed to be as user friendly as possible for novices. It basically lets us to create a realistic 3D environment in virtual reality head mounted displays (VR HMDs), as well as to build an interactive content. There is a set of steps given below to create such environment.

Steps:

1. Scaling the model that made with Sweet Home 3D and importing it to unity $3 \mathrm{D}$.

2. Applying HD textures onto the interior models so that they have realistic view.

3. Using simple shading application for creating diffuse, normal and ambient occlusion maps to create realistic 3D textures.

4. Creating and graphic user interface to change the flooring and walls textures and colors through the handheld controller.

5. Implementing teleporting system so that user can move from one room to another one easily without walking.

6. Setting light prob group and reflection group, then running baking in order to make the look realistic.

7. Finally, building the application into HTC vive VR HMDs, so that user can freely move as if he was there.

Here, a reflection probe is to capture a spherical view of its surroundings in all directions. This captured image is stored as a cube map to be used by objects with reflective materials. In addition, using light probes provide high quality lighting on moving objects in the scene (Figure 2).

Simulating global illumination accurately is challenging and can be computationally expensive (low fps), during real time rendering. For this reason, in our interactive $3 \mathrm{D}$ environments we use pre-calculation, called as baking, instead during realtime to overcome this problem. 

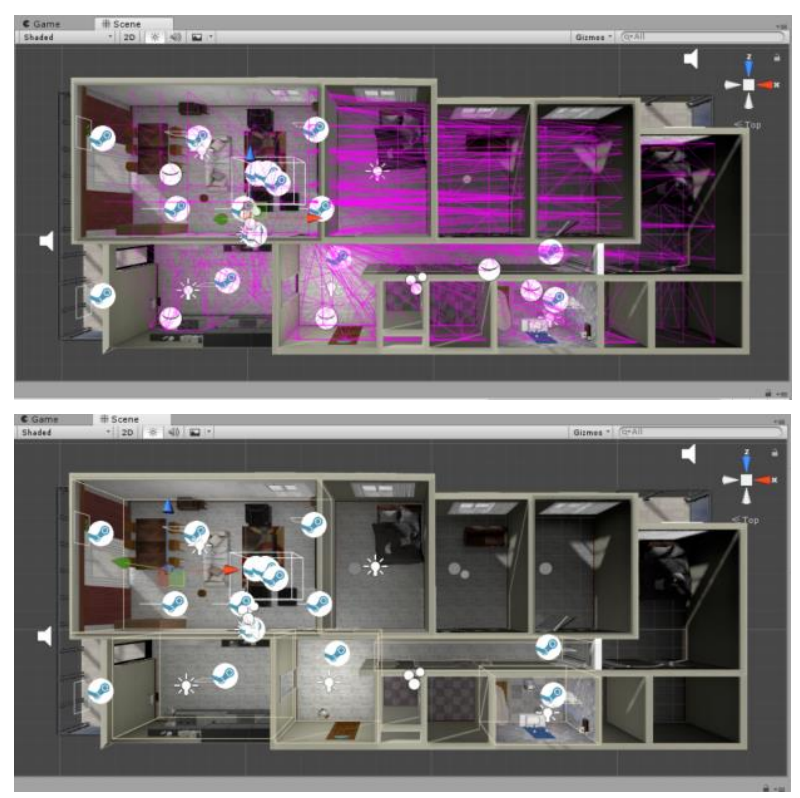

Figure 2. 2D floor plan (upper), and its 3D view with split screen in Sweet Home 3D. (bottom)

\subsection{Interaction Design}

We implemented two different types of interaction; changing materials textures such as colouring wall and floor, and teleport from one location to another within the house.
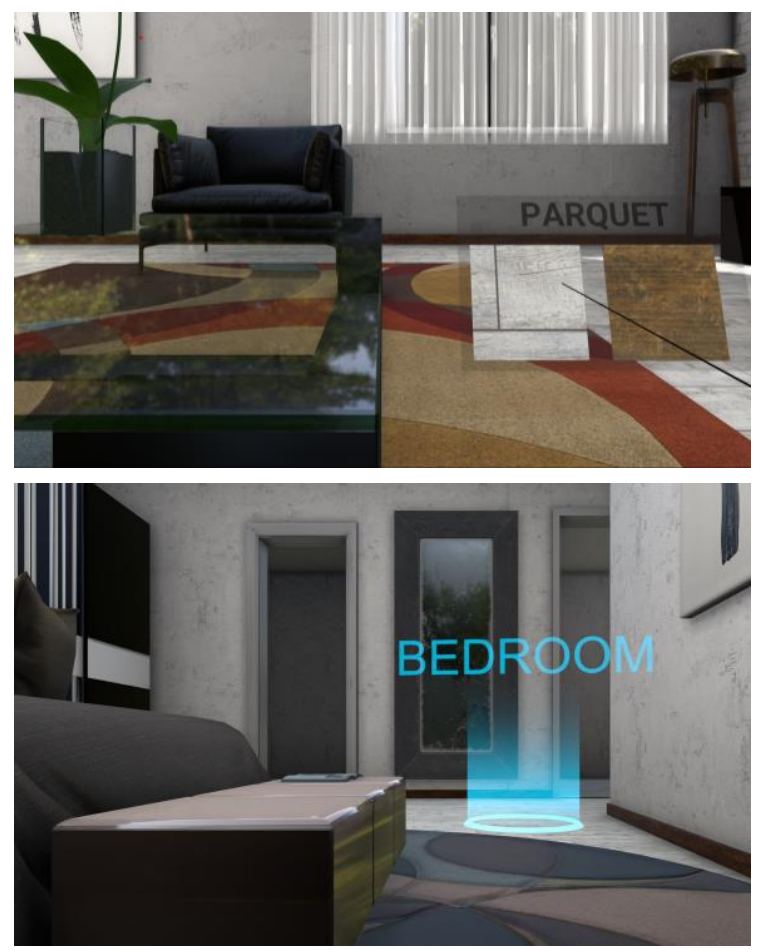

Figure 3. Graphical user interface to change material textures (upper). Teleporting point (bottom).

The graphical user interface (GUI) is appeared when the user presses the button on hand-held controller. After GUI appears the user can change the colours through a ray casted from the controller (Figure 3-upper). By casting a curative ray to a point through the hand-held controller, the user can teleport to that point (Figure 3-bottom).

\section{PRELIMINARY TESTS}

We conducted a preliminary user study to receive feedback from a group of users on our interactive real estate VR application. The experiment aimed at receiving users' opinions regarding four main criteria: presence, interface design, graphics quality, and persuasiveness.

18 participants ( 5 female) volunteered, ages 21-42 (mean 24.8, SD 5.95). Three participants had previous experience with head-mounted displays. Participants wore HTC vive HMD with a hand-held controller and freely walked in $4 \times 3 \mathrm{~m} 2$ interaction area. They were asked to freely observe the interior, modify the walls and floors, and teleport to observe other rooms of the entire house. After the experiment, participants were asked four questions, which can be seen in Figure 3, regarding the experience.

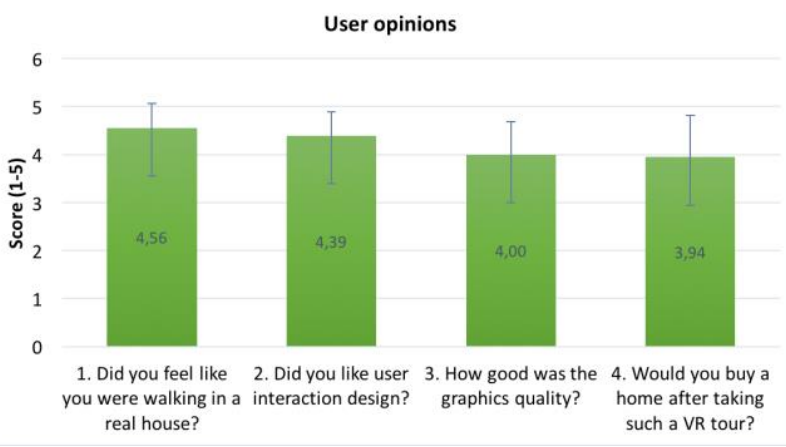

Figure 4. Users' score regarding VR real-estate interaction

Participants generally liked the way of interaction and the graphics quality, felt like they were in a real house, and wanted to buy a house after taking such a tour (Figure 4). Two participants mentioned that using arrow key could be better instead of teleporting.

\section{CONCLUSION}

Today, the tendency of property investors is to purchase a property off-the-plan as they can buy it cheaper than its real value. However, purchasing a property off-the-plan is problematic for the investors since they cannot imagine the completed format of the property. Therefore, in this study, we have created a real estate-purposed low-cost 3D interactive VR application that visualizes the interior architecture of the property. By doing so, the property sellers do not have to construct a demonstrative real property for advertising purpose. Therefore, it saves money and time for the sellers, buyers.

Instead of using highly resource consuming and specialist required software such as SketchUp, 3ds Max, Blender, SolidWorks, we used the easy to use, lightweight and free software Sweet Home 3D and Unity. Former extracts 3D interior design model of the building from the 2D floorplans with a novice-friendly interface, latter enables us to create a fully interactive 3D virtual environment. The feedbacks from the preliminary study shows that our real estate-purposed VR application generally satisfies the expectation of the property investors. They generally feel themselves in a real property since our application presents high-quality realistic interior 
architectural design. However, some of participants mentioned that it would be better in terms of presence if they moved through direction keys instead of teleport interaction.

\section{REFERENCES}

Bogdan-Alexandru Deaky, Luminita Parv, 2017, Virtual Reality for Real Estate, Experiment@ International Conference, 2017, University of Algarve, Faro, Portugal, June 6-8.

Petar Pejić, Sonja Krasić, Milica Veljković, Srđan Sakan, Taško Rizov, 2017, Augmented and Virtual Reality Application in Traditional Architectural Project Presentation - Case Study of "MH Petra" House, FME Transactions, (45)2, pp. 229.

Sanni Siltanen and Virpi Oksman, 2013, User-Centered Design of Augmented Reality Interior Design Service, International Journal of Arts \& Sciencesi, 6(1), pp.547-563, ISSN: 19446934.

Prabhakar Mudliyar, Yuvraj Ingale, Sanket Bhalerao, Onkar Jagtap, 2014, Virtual Reality for Interior Design, International Journal of Research in Advent Technology, (2)3, E-ISSN: 2321-9637.

Xiao Yu, 2011, Research and Practice on Application of Virtual Reality Technology in Virtual Estate Exhibition, Procedia Engineering, 15, pp.1245-1250.

Spyros Vosinakis, Philip Azariadis, Nickolas Sapidis, Sofia Kyratzi, 2007, A Virtual Reality Environment Supporting the Design and Evaluation of Interior Spaces, 4th Intuition Conference on Virtual Reality and Virtual Environments, Athens.

Shaoliang Qi, Virtual Interior Design Based On VRML AND JAVA, 2012, International Conference on Medical Physics and Biomedical Engineering, Physics Procedia, (33), pp.16141620. 